\title{
DETERMINATION OF THE ORIENTATION DISTRIBUTION FUNCTION FROM ARBITRARY DIFFRACTION DATA
}

\author{
J.A. Supunar \\ Mining and Metallurgical Bngineering \\ McGill University, University St. \\ Montreal, CANADA H3A 2 A7
}

\section{ABSTRACT}

A method proposed by Bunge in which we assume a fit between the theoretical ODF and the isolated experimental points was used to calculate the ODF from experimental data chosen in an arbitrary way in a pole figure and an inverse pole figure space. In particular, this technique was used to obtain the ODF from the measurements of three, two and one pole figures, from the data obtained using the reflection technique only or transmission technique only, from inverse pole figures and from the photo showing the Debye-Scherrer rings. Various tests described in this paper allows us to suggest an empirical rule indicating that the number of experimental data points should be three times higher than the number of required series expansion coefficients of the ODF. In addition, other factors, for instance, the strength of texture and the distribution of experimental points are also important.

\section{INTRODUCTION}

The X-ray methods commonly used in texture laboratories require texture measurements made using the reflection and the transmission method. Unification of the results of these two techniques always introduces a considerable error. Nevertheless full pole figures are required to calculate by integration the series expansion coefficients of the pole figures; this allows to solve a system of equations relating the coefficients of expansion of the pole figure and the ODF coefficients'. Since the measurements of the back reflection part of the pole figure does not require a time consuming preparation of the specimen, it has been proposed by Morris ${ }^{2}$ and also by Jura and Pospiech that this back reflection pan alone can be used to determine the ODFs coefficients by integration over an available interval of the pole figure. In this case, the orthogonality relations of the surface spherical harmonics will not hold and thus the solution of $C_{l}^{\mu v}$ results in a large system of equations containing all pole figure coefficients. The method therefore offers a simpler experimental procedure at the expense of more complex computation. Bunget has further relaxed the experimental requirements, suggesting that the procedure does not need integration at all but requires a least squares fit between the theoretical ODF and the isolated experimental points.

$$
\sum_{i} \sum_{j}\left[P_{i j} N-P_{i j}^{\mathrm{th}}\right] W_{i j} \Rightarrow \min
$$

Where $P_{y}$ is measured at point $i$ (having angular coordinates $x_{,}, \beta$ ) of the pole figure $j, N j$ is a normalizing factor used to express the pole density value in units corresponding to random density, and $W_{14}$ is a weight factor.

A theoretical pole figure is expressed by the following formula

$$
P_{i j}^{\mathrm{th}}=1+\sum_{l} \sum_{\mu} \sum_{v} C_{l}^{\mu v} \frac{4 \pi}{2 l+1} \dot{k}_{l}^{\mu}(j) \dot{k}_{l}^{v}(i)
$$

where $C_{l}^{\mu v}$ are the series expansion coefficients of the ODF and $\dot{k}_{l}^{\mu}(j)$ and $\dot{k}_{l}^{\nu}(i, j)$ are the surface spherical 
harmonics invariant with respect to crystal and specimen symmetry. Minimization of Equation 1 is carried out with respect to variables $N_{j}$ and $C_{l}^{\mu v}$ and yields previously presented relations.

\section{EXPERIMENTAL VERIFICATION OF THE METHOD}

Using the method proposed by Bunge, we are free to select the experimental points in pole figure orientation space, however, one should remember that such a selection might affect the accuracy of ;the obtained results. In order to test the method we will compare the ODF obtained using three complete pole figures with the ODF obtained using the partial pole figures. The comparison will be based on three parameters:

(1) the number of data points

(2) the measured area of the pole figure and

(3) the maximum value of the series expansion number.

The texture of $\mathrm{Fe}-\mathrm{Si}$ transformer steel will be analyzed restricting the amount of data available for ODF calculation.

The ODF was calculated at first from three full pole figures', each having 200 data points selected at equal angular intervals. The pole figure, $(200)$, calculated from series expansion coefficients of the ODF was in fact identical as the $[200$ ] experimental pole figure (see Fig.1). Another test was made using the reflection part of the pole figures only $\left(0<\alpha<40^{\circ}\right)$. Three pole figures were used in calculation with 37 data points on each pole figure. Similarly, in the next test, the transmission data has been used alone to determine the ODF. The results obtained show that the transmission technique can provide a reliable series of expansion coefficients of the ODF, which can be used to obtain the pole figure shown in Fig.2. If one further restricts the experimental data by choosing, for example, a triangle of the pole figure i.e. $\alpha(0,90)$ and $\beta(45,90)$, limiting a number of available experimental data points to $3 \times 37$, one can still obtain sufficient accuracy of the pole figure determination (Fig.3) from ODF's series expansion coefficients. In the next series of tests, the number of pole figures used in the calculation was limited to two, with 100 points on each pole figure and, various combination of the two pole figures were tested. Finally the ODF was calculated only from one pole figure. In this later case, the values of $C_{l}^{\mu \nu}$ are satisfactory for low $l$ values, but for $l=12$ and $l=14$, high values of the $C_{l}^{\mu v}$ were obtained indicating the instability of the solution.
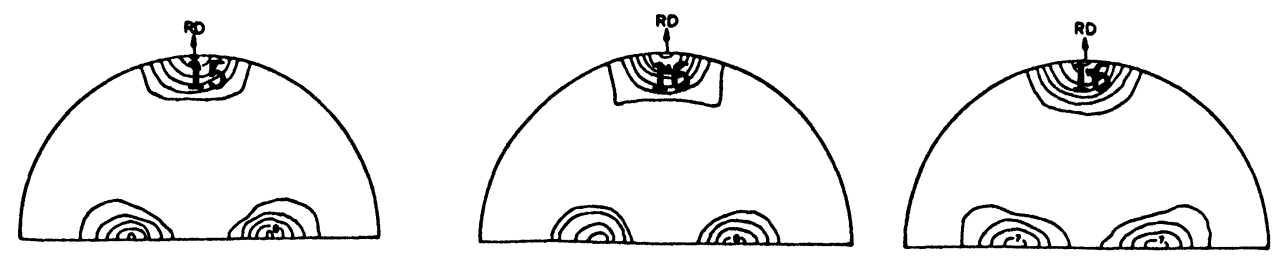

Figure $1\{200\}$ pole figure calculated from the ODF (experimental data from three pole figures were used).

Figure $2\{200\}$ pole figure. The $C_{l}^{\mu \nu}$ efficients calculated from transmission part $\left(60^{\circ}<\alpha<90^{\circ}\right)$ of the three pole figures.

Figure $3\{200\}$ pole figure. The $C_{l}^{\mu v}$ calculated from a part of the pole figure $\alpha(0-90)^{\circ}, \beta(0-45)^{\circ}$ 


\section{CALCULATION OF THE ODF FROM THE INVERSE POLE FIGURES USING ENERGY DISPERSIVE DIFFRACTOMETRY}

The energy dispersive diffractometry offers new possibilities of texture studies and some applications of these techniques have already been examined. Szpunar at al." applied the technique to determine the inverse pole figures in textured specimens, later Szpunar and Gevard' used the method for simultaneous measurements of several pole figures in Alpha and Beta Brass. Various geometries of energy dispersive texture measurements were already discussed previously by the author", specifically the geometries for on-line texture control and for the study of texture transition under the influence of external parameters. Bunge and Kopineck' have applied the energy dispersive diffractometry to obtain on-line information about texture. It was also demonstrated earlier by the author that energy dispersive diffractometry can be used to calculate the ODF. Experimental data measured on the four inverse pole figures, in 9 points on each pole figure were sufficient to obtain the series expansion texture coefficients up to $l=12$. The $[110]$ pole figures calculated from these coefficients is compared in Fig.4 with the pole figure measured using neutron diffraction ${ }^{20}$.
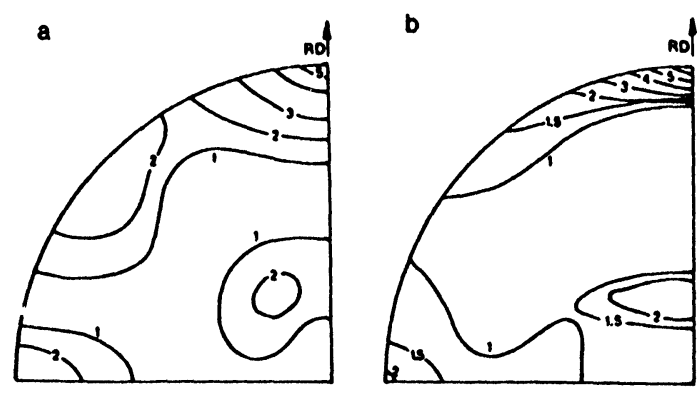

Figure $4\{110\}$ pole figure calculated from: (a) $O D F$ coefficients, $I_{m=1}=12$. (b) $\{110\}$ pole figure measured using neutron diffraction.

\section{ODF CALCULATION FROM THE DEBYE SCHERRER RINGS}

Synchrotron radiation offers a new possibility of studying the texture and the structure of polycrystalline materials. Supunar and Davies" ${ }^{11}$ have already demonstrated that it is possible to obtain reliable texture information in a few seconds using the $X$-ray film technique. The high intensity of the beam and the wide spectrum of radiation makes possible new experiments, in particular, the synchrotron radiation can be used for studying time dependent texture transformations. Such time dependent experiments, however, have to be performed using measuring systems and geometries that are quite different from traditional geometries of texture measurements. The question arises, therefore, about how to convent the new type of experimental data into the ODF.

As an example we will analyze the results of synchroton radiation $\mathrm{X}$-ray experiments reported earlier by Szpunar and Davies". Data on texture of an $80 \%$ cold rolled aluminium sheet specimen were obtained in the transmission geometry with the specimen perpendicular to the direction of the beam. The wavelength of $.99 \AA$ was selected by monochromator and the beam cross-section diameter was about $2 \mathrm{~mm}$. A two-second exposure of the film gives four diffraction rings. The relative intensity changes were recorded along the Debye-Scherrer rings (see Fig.6) and were used by Sxpunar et al. ${ }^{12}$ to calculate the ODF.

The coefficients up to $l=8$ were obtained (see Table 1 ) and obviously more experimental data would be necessary. More information about texture can be easily gathered in the synchrotron radiation experiments using a shorter wavelength. This should allow us to obtain the experimental data which are sufficient for determination of the higher order series expansion coefficients of the ODF. 
Table 1 Series expansion coefficients of the ODF

$\begin{array}{ccccccc}1 & \mu & \nu=1 & 2 & 3 & 4 & 5 \\ 4 & 1 & -4.493 & 0.154 & -0.653 & & \\ 6 & 1 & 14.902 & -0.009 & 0.260 & 0.240 & \\ 8 & 1 & -5.889 & -0.119 & 0.555 & 0.100 & 0.616\end{array}$

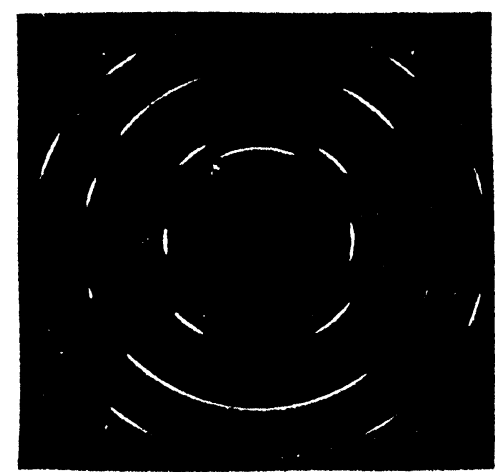

Figure 5 Diffraction rings obtained using synchrotron radiation $(\lambda=0.99 \AA)$ for textured specimen of rolled aluminium plate. Rolling direction up.

ain)

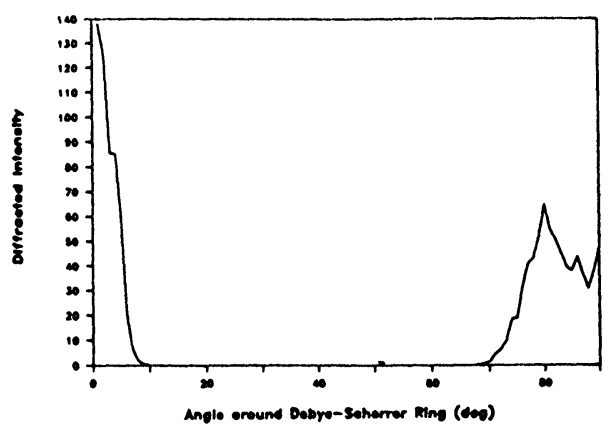

(a)

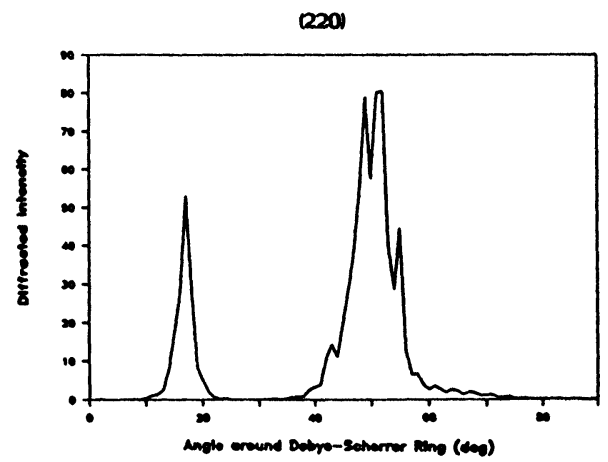

(c)

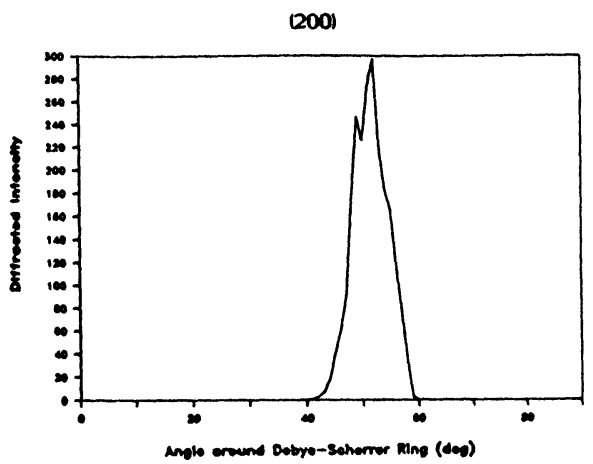

(b)

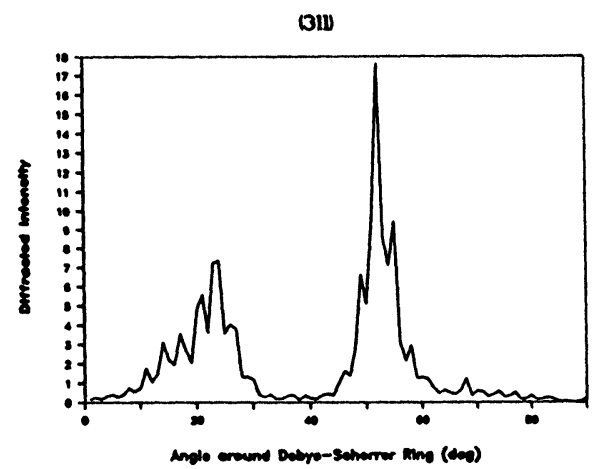

(d)

Figure 6 Variation of diffracted intensity as a function of the angle around the Debye-Scherrer ring for (a) 111 ring, (b) 200 ring, (c) 220 ring and (d) 311 ring. Angle zero corresponds to rolling direction. 


\section{CONCLUSIONS}

The method used in our analysis of texture measurements can be applied to any experimental data registered in the pole figure or on the inverse pole figure. It can be used as well to obtain fully quantitative information about texture using various non-traditional geometries of texture measurements. The number of significant experimental data points is a variable which determines how many series expansion coefficients can be reliably calculated. The accuracy of calculation depends as well on other factors such as the type and sharpness of measured texture.

Using this general method of ODF calculation we have demonstrated that the orientation distribution function can be determined from arbitrary pole figure regions, form the inverse pole figures and the intensity of DebyeScherrer rings obtained in a synchrotron radiation experiment.

\section{REFERENCES}

1. H.J. Bunge, Texture Analysis in Materials Science, Butterworths, London, 1982.

2. P.R. Morris, Adv. X-ray Anal. 18, 514, (1975)

3. J. Pospiech and J. Jura, Z. Metallkde 65, 324, (1974)

4. H.J. Bunge, Texture of Crystalline Solids 2, 169, (1977)

5. J.A. Szpunar and D.C. Hinz, J. Mat. Sci., 25, 2846, (1990)

6. J.A. Szpunar, M. Ojanen and E. Laine, Z. Metallkde, 65, 221, (1974)

7. J.A. Szpunar and L. Gerwand, J. Mat. Sci., 15, 469, (1980)

8. J.A. Szpunar, Texture of Crystalline Solids 4, 171, (1981)

9. H.J. Bunge, H.J. Kopineck and F. Wagner, Textures and Microstructures 11, 261, (1989)

10. J.A. Szpunar, Textures and Microstructures 12, 243, (1990)

11. J.A. Szpunar and S.T. Davies, Proc. Seventh Conf. on Texture of Materials, 613 (1984), Amsterdam, Netherlands, Society for Materials Science.

12. J.A. Szpunar, P. Blandford and D.C. Hinz, J.Appl.Cryst., 22, 559, (1989) 